\title{
COMUNICACIÓN Y MÚSICA. Campos de tensión en la construcción de una industria creativa y cultural en el Oriente Antioqueño - Colombia
}

\author{
Carlos Andrés Arango* \\ Berónica Rojas Alzate** \\ DOI: https://doi.org/10.33571/revistaluciernaga.v11n22a6
}

\section{Resumen}

En el artículo se presenta la reflexión producto de investigación en torno a las industrias creativas y la percepción que de ésta se tiene en el Oriente antioqueño, específicamente en el Municipio de Marinilla y El Carmen de Viboral. Se evidencian campos de tensión en torno a la vocación vs profesión; vaivenes políticos vs interese colectivos; cultura, talento y gestión local vs requerimientos globales; optimismo por la industria musical vs bajo consumo de esta en dicha zona.

El paso de la vocación autoexpresiva en la música a un desempeño profesional en el marco de una industria creativa o cultural, demanda un sinnúmero de saberes y prácticas para los que se requiere formación y experiencia.

Palabras clave: Industrias creativas; industrias culturales; música; comunicación.

Recibido. Julio 22, $2019 \quad$ Aceptado. Agosto 05, 2019

** Comunicación Social, Universidad Católica de Oriente. Integrante del semillero Música y Comunicación. Gestora cultural en el ámbito de la música. En la investigación fue responsable del rastreo y fichaje bibliográfico, la elaboración y transcripción de las entrevistas, así como su primer análisis para efectos del presente artículo. Ambos autores participaron activamente en la discusión

de los resultados del trabajo, en la revisión y aprobación de la versión final del trabajo. e-mail: beronicarojasalzate.13@gmail.com 


\title{
CREATIVE INDUSTRIES: MUSIC AS VOCATION AND PROFESSION
}

\author{
Carlos Andrés Arango* \\ Berónica Rojas Alzate** \\ DOI: https://doi.org/10.33571/revistaluciernaga.v11n22a6
}

\section{Abstract}

The article presents the reflection resulting from a research into the creative industries and the perception of this in the East of Antioquia, specifically in the municipalities of Marinilla and El Carmen de Viboral. Stress fields are evidenced around the professional calling VS. profession; political fluctuations VS. collective interests; culture, talent and local management VS global requirements; optimism for the music industry VS. low consumption of this cultural representation in that zone.

Key words: Creative industries; cultural industries; music; communication.

Received. July 22, 2019

Accepted. August 05, 2019

*Communicator, master degree and PhD candidate. Leader of the Communis research group, professor-researcher in Social Communication, Universidad Católica de Oriente. Areas of study: music, urban imagery, communication and culture. As main author he consolidated the bibliographic and interview analysis, the contextualization and the final writing of the work. Orcid: http://orcid. org/0000-0002-2120-3304; e-mail: carango@uco.edu.co

** Social Communication, Universidad Católica de Oriente. He is a member of the Music and Communication seedbed. Cultural manager in the field of music. In the research, he was in charge of the tracking and bibliographic recording, elaboration and transcription of the interviews, as well as their first analysis for the purposes of this article. Both authors actively participated in the discussion of the results of the work, in the review and approval of the final version of the paper. e-mail: beronicarojasalzate.13@ gmail.com 


\title{
COMUNICAÇÃO E MÚSICA. Campos de tensão na construção de um indústria criativa e cultural em Oriente Antioqueño - Colombia
}

\author{
Carlos Andrés Arango* \\ Berónica Rojas Alzate** \\ DOI: https://doi.org/10.33571/revistaluciernaga.v11n22a6
}

\section{Resumo}

O artigo apresenta a reflexão resultante da pesquisa sobre as indústrias criativas e a percepção disso no Leste de Antioquia, especificamente nos municípios de Marinilla e El Carmen de Viboral. Os campos de tensão são evidentes em torno da vocação versus profissão; altos e baixos políticos versus interesse coletivo; cultura, talento e gestão local versus exigências globais; otimismo para a indústria musical versus baixo consumo da mesma nessa área.

A passagem de uma vocação auto-expressiva na música para uma actuação profissional numa indústria criativa ou cultural exige uma grande quantidade de conhecimentos e práticas para as quais é necessária formação e experiência.

Palavras chave: Indústrias criativas; indústrias culturais; música; comunicação.

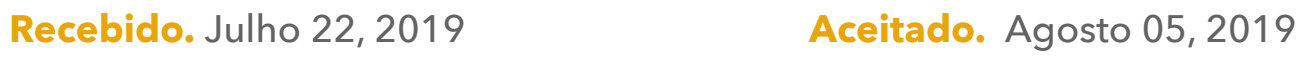
e cultura. Como autor principal, ele consolidou a análise bibliográfica e a análise de entrevistas, a contextualização e a redação final da obra. Email: carango@uco.edu.co.http://orcid.org/0000-0002-2120-3304; Email: carango@uco.edu.co

** Comunicação Social, Universidad Católica de Oriente. Ele é um membro do viveiro de Música e Comunicação. Gestor cultural na área da música. Na pesquisa, foi responsável pelo acompanhamento e registro bibliográfico, a elaboração e transcrição das entrevistas, bem como sua primeira análise para os fins deste artigo. Ambos os autores participaram ativamente na discussão dos resultados do trabalho, na revisão e aprovação da versão final do trabalho. e-mail: beronicarojasalzate.13@gmail.com 


\section{Introducción}
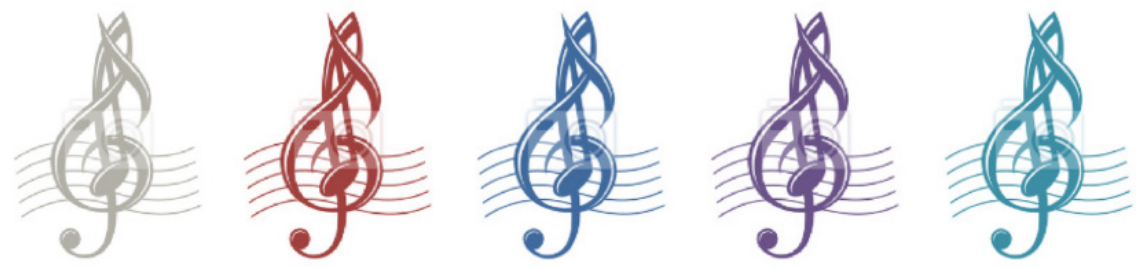

Desde que John Howkins propuso el concepto de economía creativa (Ghelfi, 2005), muchas han sido las reacciones que se han generado. La advertencia servía para señalar que en el intercambio económico mundial, un importante componente se derivaba de productos simbólicos para cuya creación no se requiere, como en las economías industriales, de complejas redes de maquinaria. El foco de esta economía estaría en la creatividad. Para países con dificultades técnicas o financieras para la configuración de un avanzado sistema industrial, esto abriría puertas a la competitividad en el escenario global, toda vez que la creatividad es un bien para cuya adquisición no se requiere otra cosa que talento humano.

Orientado hacia ese concepto, Buitrago y Duque (este último, actual presidente de Colombia), escribieron, desde el Banco Interamericano de Desarrollo, un libro que terminaría siendo el marco referencial de la política colombiana en la materia, con el particular nombre de Economía naranja (Buitrago y Duque, 2013). En tanto dicha orientación es política pública, los debates se concentran en torno a su capacidad de materialización concreta en el territorio nacional. No deja de preocupar, que a medida que se verifica la desaparición de empleos estables, contratos a término indefinido y, en general, garantías para los empleados, se impulsan iniciativas como el emprendimiento bajo premisas como la economía naranja.

Bauman (2007) indica que una de las razones por las cuales se transita de la modernidad sólida a la líquida (posmodernidad, o modernidad tardía) es por la desregulación de asuntos como la cultura y el empleo, habida cuenta del desinterés de los Estados por seguir constituyéndose como gobiernos de bienestar. Remedios Zafra (2017) señala que, si bien la cantidad de jóvenes recién egresados de las universidades cada vez encuentran más alternativas de poner sus dones y talentos al servicio de su proyecto de vida, la monetización de los mismos es un reto que tan sólo unos pocos logran superar. Como agravante, se encuentra que al dejar a la cultura sometida a los juegos de mercado, algo propio de las sociedades de consumidores, los gigantes de la industria son la competencia directa de individuos que, aislados, buscan obtener ingresos con sus blogs, la venta de guiones que, crédulos, entregan a agentes de la industria cinematográfica, - aún mediante la publicación de sus fotografías en plataformas que, mediante diferentes modelos de negocio, generan una monetización complicada para los creadores de contenido (Carrasco y Saperas, 2011).

La pregunta por las industrias creativas, visto en el contexto de una región en proceso de urbanización (Montoya, 2016), reporta al menos, tres situaciones de contexto que se deben considerar. En primer lugar, la innegable cantidad de talento surgida desde sus municipios, cuyo legado para la historia se muestra en compositores, pintores e intérpretes musicales (Castro y Quintero, 2018). Así por ejemplo en El Santuario, un municipio vecino de los dos territorios objeto de estudio en este trabajo, nació el compositor Roberto Pineda Duque (1910-1977), quien se toma en la historiografía musical como un abanderado del vanguardismo en Colombia. Aún el nombre de Sixto Arango 
Gallo (1916-1985), destacado compositor de música religiosa, es un referente clave en el movimiento cultural del Oriente antioqueño.

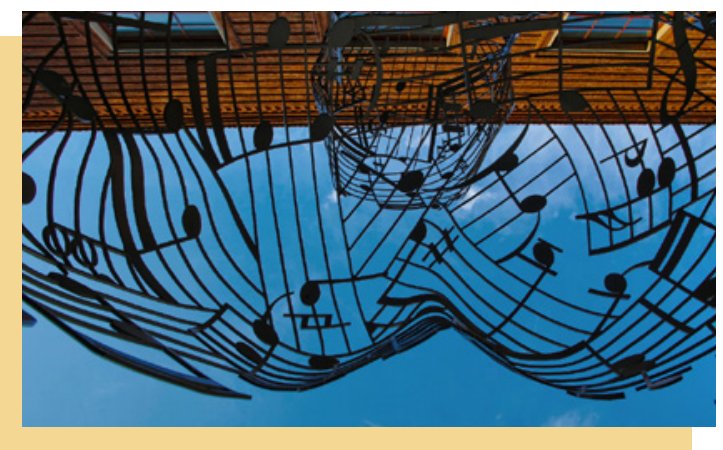

En segundo lugar, las políticas públicas, cuando se observa la postura de los entes municipales en torno a la cultura, se detecta la falta de políticas culturales (solo dos municipiostienen una política cultural clara). Pero, además, al revisar estos documentos, se aprecia una lectura bastante vinculada a la cultura como manualidades, artesanías o formas de expresión, desde las que no se pretende otra cosa que permitir vocaciones individuales aisladas. Es decir, tanto si se lee como (alta) cultura, o como folclore, los planes representan aquella visión en la que la cultura es un bien prescindible, un cierto objeto de lujo. Por eso, no se avizoran estrategias que la proyecten a otros escenarios, más allá de la idea misma de auto-expresión.

En tercer lugar, el creciente proceso de urbanización que vive la región, hace que referentes globales se integren a las formas de vida de las personas, asunto que invita a los creadores a iniciar sus procesos artísticos con miras a formas, estilos y códigos culturales que circulan por redes de intercambio a los que les resulta complicado ingresar. La utopía de internet hace pensar que basta con subir contenido para ser reconocido en un ámbito superior al local, pero la realidad muestra que no es tan sencillo como publicar videos en Youtube o abrir cuentas en Spotify (Capurro, 2014).

La suma de esos tres agravantes hacen que, para el caso de la música, primero, los artistas de referencia para los nuevos creadores no pueden ser los pertenecientes a otras generaciones, pues su obra trascendió en épocas y estilos musicales diferentes; segundo, que no se cuenta con políticas culturales claras en los municipios (nada que trascienda la enseñanza musical, los conciertos, y los apoyos a bandas sinfónicas, de rock o conjuntos de cuerdas); tercero, que mientras las redes sociales electrónicas parecen un escenario ideal para la difusión del propio material, las estrategias de conversión de esta visibilidad en un proyecto de vida que encuentre sustento económico aún no aparecen.

Bajo este contexto, se parte de la premisa de que la tensión entre industrias creativas e industrias culturales pone a prueba percepciones que movilizan tanto los investigadores como los partícipes de la industria creativa. Sin embargo, debido a la evidencia de la cantidad de personas que producen bienes culturales, músicos en este caso, revisar la dinámica del sector permitirá trazar lazos de comunicación que mejoren las interacciones entre los componentes de éste. Como lo señalan Banks y O'Connor (2009):

Las críticas a las industrias creativas se pueden resumir en las siguientes apreciaciones: constituyen un relato economicista de la cultura; contienen incoherencias de definición con las consiguientes consecuencias para la obtención de datos y la realización de pruebas; idealizan el trabajo 'creativo' y, sobre todo, su adaptación fuera del país que las originó constituye un imperialismo implícito por la exportación del concepto y de su implantación real a escala mundial (p. 50).

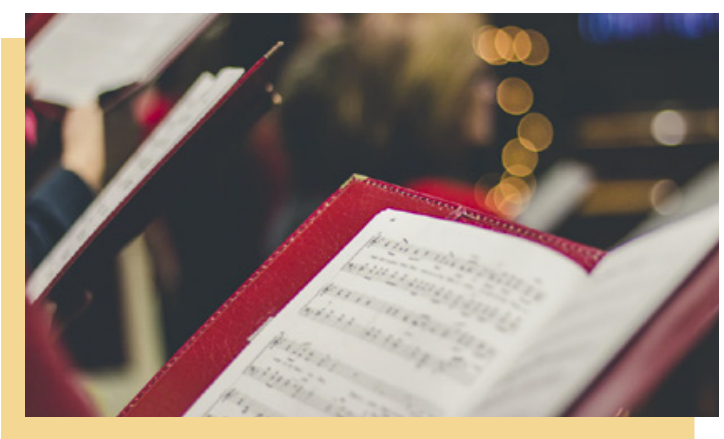




\section{Metodología}

Se realizó una investigación exploratoria, por considerar que dicho nivel de aproximación era el más adecuado para propiciar un primer encuentro con el problema de investigación (Cazau, 2006). Por tanto, se recurrió a dos repertorios de información, para ambientar los avances de la literatura en el panorama real de lo que se vive en los territorios estudiados. Uno denominado literatura científica y otro llamado fuentes primarias (artistas y promotores activos en los territorios de interés, así como usuarios de las ofertas culturales musicales).

La revisión en bases de datos, permitió el acceso a las reflexiones que en los últimos años ha suscitado el tema de las industrias creativas; con las fuentes primarias se reconoció la percepción de creadores, historiadores y población de los municipios, mediante entrevistas en profundidad. El propósito de este planteamiento metodológico fue contrastar dos niveles de información: el documental con el experiencial. Las muestras se recolectaron, en el caso de la literatura científica, mediante los filtros de relevancia, impacto y vigencia; en el caso de las entrevistas, tres personas por cada rol, en cada municipio; para ello se siguió un muestreo por conveniencia (cercanía a los sujetos y reconocimiento de su papel en el contexto cultural de los territorios, esto último particularmente en historiadores y creadores).

Como se indicó en párrafos anteriores, el propósito de este diseño fue validar una primera fase de acercamiento a los actores mencionados, a partir de lo que se halló en la revisión de literatura. No se trata de un estudio concluyente, sino de una fase de exploración que buscó validar los diseños de entrevista a profundidad. Eso se hace siguiendo los principios de la teoría fundada, cercana al interaccionismo simbólico (Strauss), según los cuales, cada fase de entrevistas va acotando las referencias del marco referencial en entrevistas más cerradas, y así sucesivamente en cada fase de éstas.

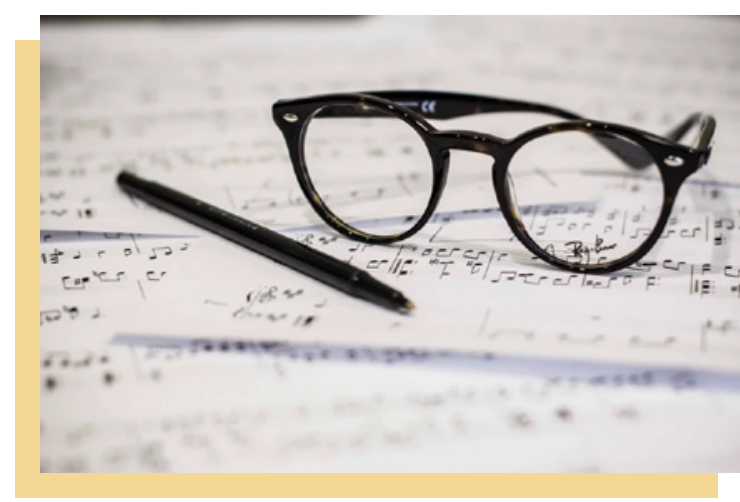

\section{Resultados}

\subsection{Revisión del concepto}

El concepto industrias creativas se comprende como el conjunto de prácticas culturales basadas en el talento creativo y artístico de las personas, pero llevado directamente al sector económico como un trabajo real que hace parte de una dinámica que aporta económicamente al territorio (Prieto Herrero, 2011).

En este sentido, los bienes y servicios culturales se producen, reproducen, conservan y difunden a partir de criterios industriales, con un foco en lo económico, pero donde lo cultural -"inmaterial", intangible, producido por un autor-se mantiene como principio activo y significativo del bien. Esta dualidad cultural y económica es lo que distingue a estas industrias de otras mercancías, y es el punto de foco de los Estados para construir las políticas culturales (Szpilbarg y Ezequiel, 2014, p. 106).

Las industrias creativas han generado la aparición de un lenguaje, de una forma de referirse a los bienes inmateriales, producidos por la creatividad humana, donde se reconoce la cultura como forma de vida en sí misma, pero con una visión más amplia que las artes tradicionales. Ello implica un cambio en el pensamiento político sobre cultura. Cunningham, cita a Wyszomirski (2008) para señalar que:

"[...] La aparición de un lenguaje al mismo tiempo más centrado en la cultura como una forma de 
vida en sí misma pero mucho más amplia que las artes tradicionales ha provocado un cambio importante en el pensamiento político pasando de ser "un sector dañado por los costes, pobre en recursos y necesitado de subvenciones" a una serie de activos comunitarios capaces de impulsar el desarrollo local" (Cunningham, 2008, p. 203).

Por lo tanto, las industrias creativas, refieren a un campo nuevo y por explorar, pero que siempre ha estado en la sociedad y ha hecho parte del ser humano: "Se sitúa a las industrias creativas o culturales como heraldos o catalizadores de algo nuevo, 'que está ahí', que exige reconocimiento, investigación y promoción; algo que señala hacia lo real, lo urgente, lo emocionante" (O'connor, 2011, p. 26). También se reconoce que abordar y desarrollar más esta industria es una necesidad urgente, pues la sociedad avanza hacia escenarios laborales diversos (Bauman, 2007), donde las personas están menos dispuestas a sufrir las consecuencias negativas de separar sus intereses, talentos y pasiones, de la forma como generan sus ingresos. Es decir, cada vez hay menos tolerancia para aceptar que las pasiones personales van por un lado y las actividades laborales van por otro.

El reto consiste, justamente, en cómo convertir esas nuevas formas laborales basadas en quehaceres artísticos, valores de libertad (de expresión y sustento económico), en un proceso de sociabilidad y desarrollo. En otras palabras, el reto consiste en que quienes deciden fomentar sus talentos artísticos no queden relegados de la dinámica económica. "La cultura no sólo se acerca a la economía, sino que es reapropiada como una herramienta o recurso político" (Szpilbarg y Ezequiel, 2014, p. 106). De hecho, Sosnowski (1999) ve a la cultura vinculada al desarrollo social, por lo cual se requiere atender las necesidades que surgen desde ella por ser un ámbito donde se negocia la experiencia, en medio de un sistema de creencias y valores (Sosnowski, 1999).

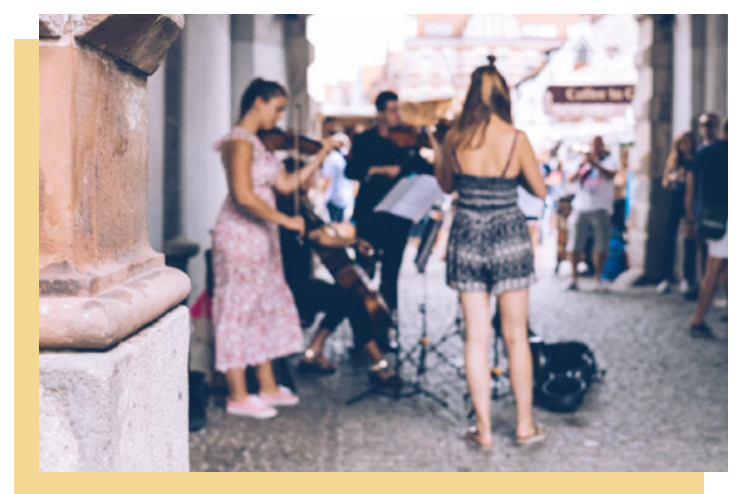

Las industrias creativas no se deben separar de los procesos culturales, por más que a partir de la teoría crítica, la fusión de estos dos conceptos causaba, cuando menos, perspicacias (Szpilbarg y Ezequiel, 2014). Sin embargo, la forma en que se marca la diferencia en el enfoque radica en comprender cómo los procesos culturales pasan a ser industria cuando se monetiza la creatividad y lo artístico. Mientras ésta, tradicionalmente entendida, circula por ámbitos de lo popular y lo masivo (Martín Barbero, 1998), los productos construidos con una intencionalidad mercantil, también entran a hacer parte de ese conglomerado de significaciones sociales que, de forma un tanto ambigua, se denomina "cultura". Mantener la separación entre ésta y la industria no hace otra cosa que impedir una adecuada profundidad para comprender los fenómenos sociales de construir, difundir, compartir y renegociar significados.

Ésto plantea, un reto particular en el horizonte de expresiones culturales, autóctonas, a través de las cuales han circulado, históricamente, valores, significados e imaginarios que hacen parte constitutiva de las identidades, particularmente en los ámbitos locales. Pero lleva también a reconocer que, desde su naturaleza misma, ni la identidad ni la cultura son bienes estáticos, pues surgen en el contexto de intercambios simbólicos de los cuales las sociedades, con distintas dinámicas y políticas de gestión, siempre han sido protagonistas (Bauman, 2002; Martín Barbero, 1998).

Así que en lo referido a "cultura" y a "industria cultural", es importante recalcar 
esa relación entre cambios en el concepto y variación en la gestión organizativa que se va evidenciando en distintos lugares del mundo:

A partir de la mutación que ha sufrido el concepto al compás de procesos sociohistóricos, se conformó un pensamiento acerca del sector cultural como una esfera económica que estaría no sólo destinada a cumplir una importante función en la economía, sino que sería central en el desarrollo de las culturas de los países. El concepto pretende ser recuperador de los aspectos culturales de las sociedades en el contexto de la llamada globalización (Sassen, 2007, p. 100).

Como indica Navarro Yáñez y Guerrero Panal (2012), el sector cultural configura un componente álgido en el viraje hacia las sociedades postindustriales, con lo cual constituye un sector fundamental en la ruta de desarrollo de las ciudades en términos de su (potencial) competitividad. Aunque este sector ayuda a las economías locales, también genera cierta competitividad respecto a las posibilidades laborales y el desarrollo más eficiente de esos procesos, asuntos que se presentan con desigualdades en un territorio u otro, lo que implica una producción y posibilidades laborales más amplias que llevarían a una posible migración de artistas desde unas localidades hacia otras.

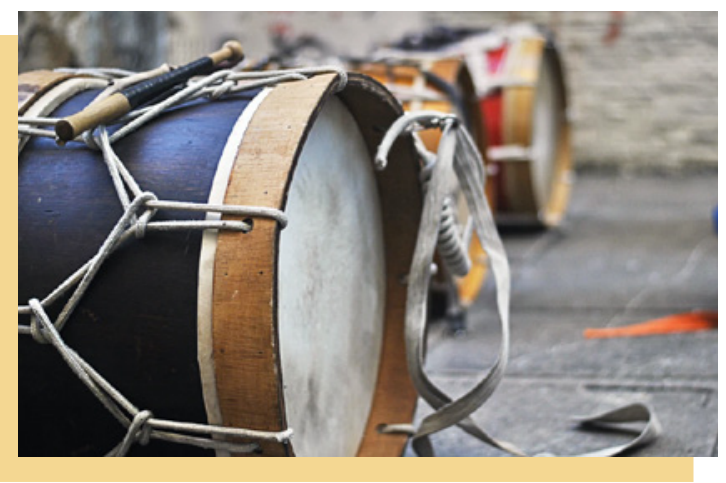

Si bien una mirada tradicional invitaría a la idea de que un artista debe permanecer en su territorio para producir desde allí su material, fenómenos como la globalización implican que, necesariamente, el entorno de producción y consumo de contenidos culturales rebasan las fronteras imaginadas anteriormente. Cuando se miran producciones en ámbitos locales, es cada vez más difícil encontrar referentes o, más bien, éstos se mezclan con referentes internacionales, lo cual va tanto en el mensaje que se construye, como en los procesos de creación y las plataformas de divulgación (Arango y Correa, 2018).

Cómo entender, de un lado, la separación industria/cultura, y, de otro, la circulación de mensajes en los ámbitos locales/ globales, si -de entrada- lo local se expresa esencialmente como un ámbito de interacción física, mientras que lo global (asociado fundamentalmente a información que circula desde y hacia casi todos los lugares del mundo) ofrece unos referentes amplios y que tienden a la estandarización (mainstream) (Martel, 2011).

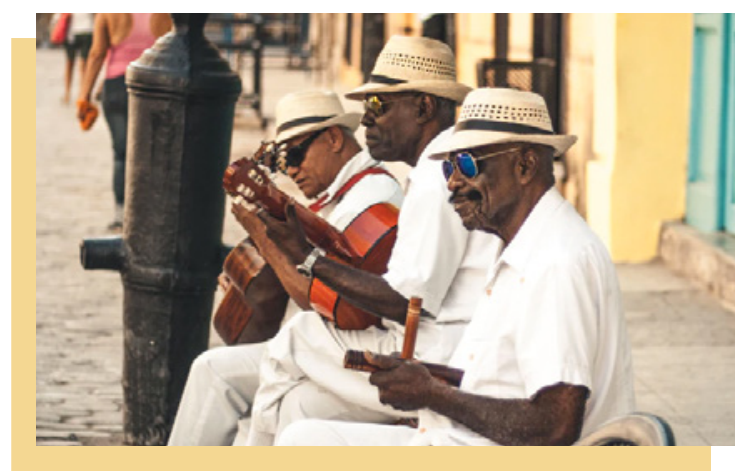

Básicamente, este sector aparece como un elemento potencial para articular el desarrollo económico y cívico de las ciudades, o más en general, de los territorios. Sobre todo, en el marco del ya constatado declive de los sectores industriales clásicos y en el cambio hacia una nueva economía enfocada en la creación, la "economía creativa (Scott, 2004, p. 73).

De hecho, en ciudades como Buenos Aires, Barcelona o Cali, las industrias creativas representan uno de los sectores actuales más influyentes en su economía, puesto que permiten al ser humano desarrollar sus habilidades y competencias creativas y 
comprender que su talento artístico tiene valor dentro del mercado, pero sin quitarle el valor identitario dentro del sector cultural (Montoya Restrepo et al, 2016). Más allá de esto, incluso, experiencias como Las Vegas, Medellín o París, son muestra de cómo las marcas de ciudad trascienden el ámbito local y se elevan hasta lo global, bien intencional o espontáneamente, de forma que sus nombres (ahora marcas) logran su ingreso al imaginario global como un conjunto de significados que envuelven música, historias e imágenes del arte.

En México, "La industria creativa [...] muestra una tendencia de crecimiento, principalmente en el tema económico. Con éstas surgen nuevos tipos de empleos y trabajadores" (Castañeda-Rivera y Garduño-Bello, 2017, p. 119). Estos procesos han impulsado un cambio hacia el reconocimiento del trabajo de otros; las nuevas formas de empleo que nacen con la vocación artística como una fuente de sustento, dinamizan sectores de creación de valor que habían estado activos, en circuitos independientes, universitarios y underground, pero ahora con un impulso económico importante.

"Los nuevos tipos de prácticas culturales en todo el mundo, interesadas en crear nuevos espacios de posibilidades y colaboración, pueden ser vistas como parte del trabajo para inventar nuevas formas de colectividades sociales" (O'connor, 2011, p. 45).

Las industrias creativas han permitido reconocer y vivir los territorios de nuevas maneras y con mayor apropiación, sin diferir con el otro; por el contrario, han intentado vincularse más con los componentes del tejido social. Por eso, no es atrevido constatar que en la actualidad el trabajo en conjunto representa el potencial de estas industrias, ya que no sólo se trata de crear y vender, sino de orientar los esfuerzos con consciencia y armonía con la naturaleza, las nuevas familias y toda la diversidad.

No es gratuito que uno de los valores centrales de las economías creativas sea lo colaborativo, aspecto en el que se diferencian radicalmente de las economías tradicionales, centradas en lo competitivo (del Valle, 2011), donde los trabajadores, vinculados al sistema como entes funcionales, no veían en la obra terminada el lugar de su aporte. Por el contrario, los bienes culturales que se producen en las industrias creativas contienen altas dosis de subjetividad, y en esto hay una oportunidad inmensa, toda vez que cada ser humano es irrepetible, con lo cual su potencial creativo y productivo no tiene lo que convencionalmente se entiende como "competencia". Eso sí, se presenta el reto de visibilizar el talento como una plataforma para crear una propia voz, y esto implica habilidades especiales en dos ámbitos: relacionamiento (capacidad de construir un networking en torno al trabajo) y producción (habilidades para materializar las ideas).

En varios de los casos que se reportan como de éxito, cuando se potencializó el sector creativo también se entendió el mismo como industria y como fuente de ingresos, no sólo para los creadores sino para las regiones y las naciones. Surgió un nuevo habitus, medios culturales que, en lo urbano, servían para el aprendizaje de otras aspiraciones culturales, así como diferentes formas de vivir esas aspiraciones y llevarlas a convertirse en ingreso económico para los creadores, productores y distribuidores (Raban, 1974; Zukin, 1982).

En el encuentro de lo global y lo local, las industrias creativas promueven y revitalizan las economías locales y regionales primordialmente, resignifican la identidad y el patrimonio pues se basan en talentos humanos y en la unión de esos talentos para crear para otros "El interés creciente en este tipo de industrias es explicado en parte por la importancia de promover sectores intensivos en talento y altamente generadores de valor agregado, que además produzcan externalidades positivas en las economías locales" (Cifuentes y Millán, 2011, p. 101).

Experiencias como éstas, demuestran que el sector cultural y las industrias creativas pueden ser una potencia para el país, porque ayudan a la población 
laboralmente, a la cohesión social y a la economía interna. En ese sentido, resulta coherente trabajar y abordar experiencias locales en el Oriente antioqueño, tratando de evidenciar la realidad de estos procesos para obtener resultados adecuados sobre este sector dentro de un territorio y un contexto próximo, para arrojar luz sobre cómo se está viviendo este concepto.

\subsection{Campos de tensión pensar la industria creativa}

Se realizaron entrevistas a creadores, historiadores y usuarios de industrias creativas en los dos municipios de estudio. Siguiendo el procedimiento de microanálisis, se identificaron consensos y disensos en las percepciones expresadas por los sujetos entrevistados (Chávez Mendez, 2004). Como se trata de un primer acercamiento a la población, esta ronda permitió evidenciar los principales campos de tensión que se advierten en los testimonios de las personas entrevistadas.

\section{- Creadores- Vocación y profesión.}

Desde la noción misma de economía creativa se pone énfasis en la etapa de creación. Sin embargo, no hay que entender la creación únicamente como un proceso de expresión de talento, toda vez que asuntos como la identidad (Castro y Quintero, 2018) inciden tanto en las razones que llevan a los sujetos a crear música como en la orientación que le dan a su vocación.

De forma insistente, los creadores señalan que el decidirse a hacer música es un asunto que pasa por fuertes y no siempre fáciles debates en el entorno personal, familiar y social. Aquí pudiéramos ubicar el tránsito, con un alto componente subjetivo entre la vocación y la profesión. La inexistencia de una industria, sin duda, complica las decisiones, pues deja la iniciativa profesional sometida a un cierto ámbito de incertidumbre. Destacar el reconocimiento de la ausencia de industria como tal, parece una palabra muy grande que obviamente va al ritmo de la movida cultural del sector nacional, pues cada vez se debe pensar más en hacer empresa y profesionalizar. Sin embargo, en el Oriente aún estamos entendiendo ese concepto de industria cultural o industria musical; eso aún suena muy grande sobre todo por las personas que están involucradas en el sector, productores, sellos discográficos. Aquí en El Carmen eso sigue siendo un asunto desde las ganas del artista de mostrar lo que ha aprendido a hacer [EM3].

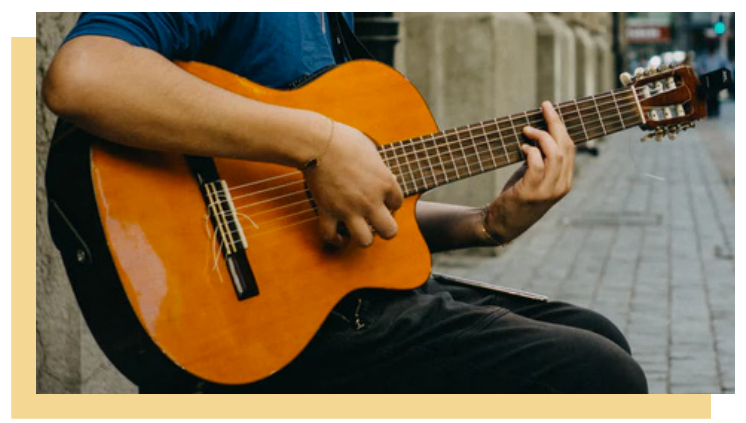

La ausencia de esa industria juega un rol paradójico. Si su existencia alimenta la esperanza de desarrollar la profesión, al tiempo reduce los ámbitos de libertad expresiva. De esta forma, el creador se juega entre dos elecciones: lamentar la ausencia de industria y su consecuente dificultad en el incremento de optar por la vocación musical como profesión, de un lado, o ver en esa falta de estructura económica una oportunidad para diseñar los caminos por los cuales transitar.

Al reconocerse la insuficiencia del entramado económico de un escenario industrial que pudiera ofrecer el Oriente antioqueño, el enfoque es externo a la región en tanto posibilidad para desarrollar la carrera y obtener mejores ingresos:

Es más fácil ser músico por fuera del Oriente antioqueño, reconocen más tu labor aunque pagan bajo ese reconocimiento y respeto por ser músico. En el Oriente hay escenarios, pero esos gestores culturales buscan llenar las tarimas con bandas reconocidas e invierten los pagos en esas bandas, es muy escaso el festival donde le paguen el valor real de un concierto a una banda local [EM3].

Por tanto, lo que se aprecia al nivel de los creadores es un desmedido balance entre 
lo vocacional y lo profesional. Las ganas de hacer música compiten con un principio de realidad que alerta sobre sus dificultades como profesión. Ésto es consistente con lo que muestran Arango y Correa (2018) en el sentido de los oficios en que se desarrollan las necesidades profesionales de los músicos: docencia musical, asistencia técnica de eventos y conciertos, bares y restaurantismo.

Los historiadores - El vaivenes de lo político vs interés de los colectivos En esta investigación se asume que, para el contexto que nos ocupa, la figura de historiador es más cercana a aquella que, en principio, aparecía como la búsqueda principal, a saber los críticos musicales. La crítica es parte fundamental del encuentro entre la oferta artística y sus públicos. Entendida en su sentido literal, sin embargo, no existe algo que se pueda denominar crítica musical en el Oriente antioqueño. Aunque existe una creciente oferta de difusión musical (en todo caso no dirigida en exclusiva a los artistas locales), el componente crítico supone una interpretación que enlaza los intereses del público con las ofertas culturales disponibles.

Por eso, la figura de los historiadores resultó clave. Salvo contados casos, en la región las personas que reciben el reconocimiento de investigadores son filántropos, voluntarios que dedican su tiempo libre a recopilar datos, estudiar personajes históricos y, en fin, participar en la invisible labor de darle un sentido temporal al acontecer cotidiano.

En lo referido a la música, lo primero que destacan los historiadores entrevistados es la cantidad de talento del que dispone la región, no solo en el campo musical, sino en todas las expresiones artísticas. Municipios como Marinilla y El Carmen de Viboral sobresalen por haber convertido esas vocaciones artísticas en parte de su oferta turística. Cuando ésto ocurre, implícitamente se da el giro hacia los procesos de city marketing (Cassián Yde, 2012), en plena consonancia con la creciente urbanización de la región. Lamentablemente, afirman, ésto ocurre más por iniciativas gremiales y privadas, que por un impulso claro desde los entes territoriales.

Con respecto al campo musical los municipios se limitan a espacios y apoyos vía becas de creación que, en todo caso, no dejan de ser acciones aisladas, un tanto coyunturales y asistémicas que, al no ser parte de políticas públicas constantes en el tiempo, no dejan de estar sometidas a los vaivenes de lo político. Por esto, es en el ámbito personal y de colectivo artístico donde se deben compensar estos vacíos.

Marinilla ha sido un plus musical en y para el Oriente antioqueño. Hemos visto como cada año nacen cantidad de músicos que contribuyen a la cultura y la identidad local, pero surgen también con la necesidad de mostrar sus procesos y vivir de su arte [EH2].

\section{- Cultura, talento y gestión local vs requerimientos globales}

Falta de gestión en plataformas virtuales. Los historiadores señalan con insistencia que si bien ha crecido la cantidad de artistas, se registra una reducción de bares y festivales, lo que implica una muy baja posibilidad de que éstos se den a conocer efectivamente. En el aspecto de circulación, reconocen la influencia de las plataformas musicales digitales y las redes sociales, pues consideran que los músicos no han sabido gestionar estos espacios.

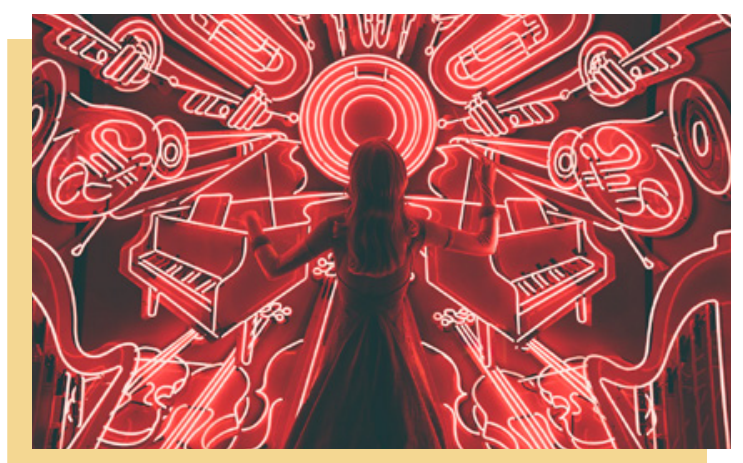

Se requiere mayor valor agregado de la oferta musical. En el entendido que las músicas se van expandiendo a estilos y prácticas de producción globales, en su presencia como artistas y como bandas en lo local no tiene algo que pueda darles, per se, un plus, un diferenciador. 
En el caso de las ofertas culturales, se aprecia que éstas van migrando por varios caminos: la elaboración de proyectos de bandas tributo (que se basan en producir covers de artistas famosos), la confección de proyectos comerciales que acogen los cánones estilísticos de músicas populares de amplia difusión nacional (ranchera, despecho...), el diseño de proyectos "de autor", a los que se llega, de entrada, con la convicción de ser iniciativas que podrán aportar mucho en lo personal pero poco en lo económico. Los dos primeros caminos aseguran la circulación por la red de eventos y bares que hay en los municipios y que se expande, con diferencias remarcables, por la región; el tercero tiene un ámbito de influencia más en los ámbitos académicos y de culto, al tiempo que exige la apertura a circuitos internacionales del world music.

\section{- Talento endógeno requerimiento exógenos}

VS

El cambio, indican, no tiene que ver tanto con el talento endógeno de las nuevas generaciones, sino a un cambio global del mundo, donde los géneros musicales, sus circuitos $y$, en general, el esquema de la cultura torna a un modelo más industrial, lo cual se traduce en una dificultad para quienes producen arte: el talento ya no es el único requisito para lograr una repercusión en el público; se requiere, además, de competencias que permitan leer el entorno cultural industrializado y sus códigos particulares. Es decir, la posibilidad de que el material circule está en relación, no solo con la calidad misma de la obra sino con habilidades de gestión para lograr que esta transite por los ámbitos requeridos.

Los usuarios. Es casi una verdad a voces que los artistas que se decantan por propuestas propias (el tercer camino identificado en las entrevistas a historiadores) no esperan en sus conciertos a nadie diferente que sus propios amigos y familiares. En cambio, los artistas que circulan con músicas y repertorios ya posicionados, si bien logran mayores ingresos, no siempre alcanzan a ser reconocidos con un nombre o una identidad propias. Esto se evidenció en las entrevistas a usuarios y resulta como consecuencia de lo que se ha venido exponiendo: al migrar hacia referentes globales en los estilos musicales, difícilmente los habitantes de la región reconocen artistas locales.

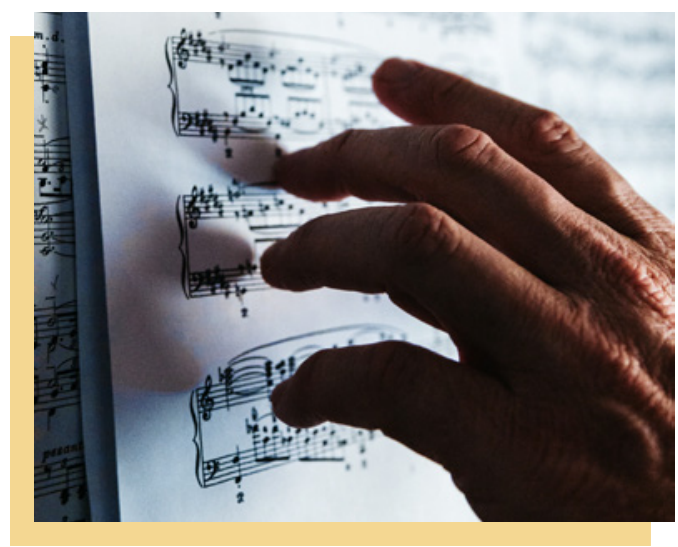

Se reconoce que la industria cultural/ musical representa un fuerte dinamismo y que hay muchos artistas trabajando arduamente y avanzando en estos procesos.

\section{- Optimismo por la industria musical versus el bajo consumo}

Se puede apreciar un cierto optimismo en esta percepción generalizada que contrasta con los bajos niveles de consumo cultural efectivo. A propósito, mencionan que, si se mira como industria, en ambos municipios falta organización y formalización en los procesos. Algunas personas consideran que es una nueva forma de generar empleos, ideas y de trabajarlas, pero que esto requiere que quienes realizan los procesos creativos para el público, comprendan la dinámica y aprendan su lugar en ella.

Otro asunto que aumenta la brecha entre las ofertas musicales y el público es su circulación a través de escenarios gratuitos y festivales, principalmente. Esto aumenta la difusión, pero desestimula la compra de productos culturales ofrecidos por los músicos. A eso se suma la poca difusión por medios como la radio o la televisión regionales, aspecto que impide una circulación amplia. De hecho, en las pocas 
ocasiones en que este material suena en las radios locales, poco se diferencia de las otras músicas programadas, de forma que es complicado reconocer a los artistas locales.

\section{Discusión}

El cercano Oriente antioqueño, específicamente en los municipios de El Carmen de Viboral y Marinilla, las industrias creativas, el sector cultural, concretamente musical, se reconoce como potencia en crecimiento, se evidencia que es un sector que tiene vitalidad dentro de ambos municipios, y son procesos que vienen resistiendo las transformaciones y exigencias que con el tiempo han surgido.

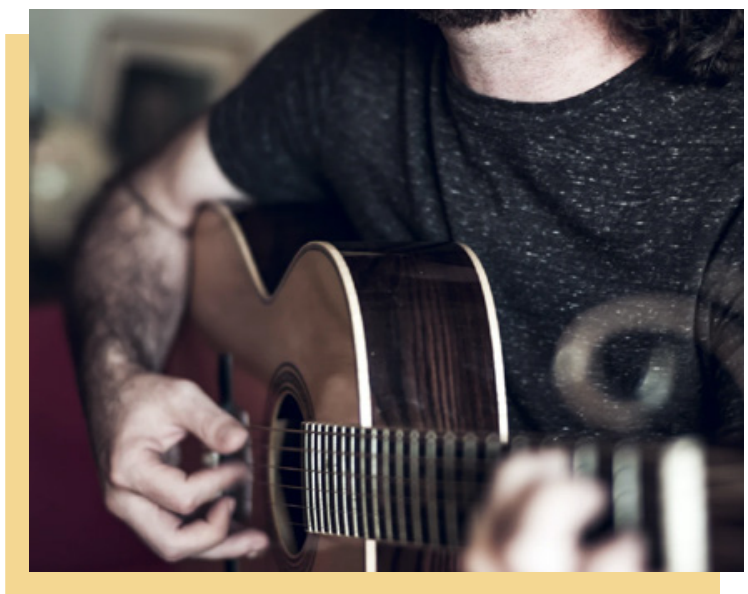

Es difícil hacer parte real de la industria musical, pues aunque el sector es fuerte en cuanto a músicos y talento, también es carente en el propósito serio y directo de convertirse en industria. Faltan espacios y cada vez se tornan más difíciles de encontrar, sobre todo en Marinilla, porque más allá de los dos festivales que realizan no hay más escenarios disponibles. En comparación, El Carmen tiene más festivales, y más espacios en bares y cafés para la presentación de los músicos. De modo que la visión y expectativas que tienen, están muy enfocadas a un desarrollo rápido y efectivo que logre ubicarlos en el mapa como reales aportantes a la industria municipal, regional y posteriormente nacional, ya que es importante consolidar a ambos municipios como potencias musicales reales.
Pero las percepciones de músicos, historiadores y usuarios coinciden que con el talento musical no es suficiente. $\mathrm{Si}$ del lado de los creadores el ingenio y la vocación permiten llegar hasta desempeños notables, sin duda es el dedicarse profesionalmente a la música lo que lleva a alcanzar nuevos niveles de interpretación, creación y producción. Ante la dificultad de convertir la vocación en profesión, los creadores migran a otros campos profesionales, al tiempo que reducen la dedicación a los proyectos artísticos en los que participan.

De tal suerte que a lo difuso de las políticas de economías creativas, en el contexto estudiado ésto aparece agravado por la falta de formación, experiencia y dominio en la parte de gestión. Los pocos casos significativos están asociados a festivales con apoyos municipales, a artistas que han ideado caminos para aumentar su circulación y profesionalizar sus proyectos, o bares que, con el tiempo, se posicionan como escenarios locales de apoyo y difusión. Casos aislados que no son producto de una red, clúster ( Valle, M., 2011) o proyecto empresarial sostenible (Jal, 2015).

Así, lo que se aprecia en el estudio es que a medida que el mundo se abre a intercambios globales, lo propiamente local se diluye, de forma que se da más fácil el ingreso de artistas nacionales e internacionales (de mayor difusión) que los propiamente locales. Ello lleva a que los creadores vean otras ciudades como el destino de sus proyectos, ante las dificultades que representa lograr que circulen en la región.

\section{Conclusiones}

Convertir los productos creativos en bienes que ingresen al intercambio económico, es una labor para la que se requiere preparación. Tal parece que las principales oportunidades se abren en el campo de la gestión de industrias creativas. El concepto se basa en la premisa de que lo único necesario es la creatividad, para que ésta se materialice en productos que impacten lo real y muevan la economía. 
Músicos, historiadores y usuarios coinciden en reconocer el talento, pero lo que conforma la textura de las industrias creativas es, justamente, su organización como industria. El tránsito del talento a productos culturales notables es uno para el que se precisan conocimientos técnicos; por tanto, el paso de la vocación autoexpresiva en la música a un desempeño profesional en ella, demanda otro tipo de prácticas que, al momento, no se pueden aprender a través de la formación (no hay quién imparta esta formación) ni de la experiencia (debido a la inmadurez industrial del sector cultural estudiado).

Todo esto parece ser consistente con el hecho de que las redes de comunicación global reelaboran los canales de intercambio, pero no siempre de manera equitativa. Sin un apoyo estatal decidido o un músculo financiero fuerte, es prácticamente imposible que se consolide algo como una industria. Dejar todo en manos de iniciativas entusiastas, pero individuales, sin la formación, la estructura política y económica clara, no hará más que alimentar el ya viejo camino de la frustración de los creadores, ratificar los estereotipos de los usuarios (según los cuales la música es lo que se ve en los medios de comunicación), y ratificar las sospechas de los historiadores: los talentos locales que tenemos por poner como ejemplos, pertenecen a épocas que ya no son interlocutoras de tiempos actuales.

\section{Bibliografía}

Arango, C. y Correa, D. (2018). Oriente visual: imaginarios de territorio en videoclips de las bandas del Oriente antioqueño. En: Desarrollo y territorio: comunidad, familia y educación. Rionegro, Fondo Editorial Universidad Católica de Oriente.

Arango Lopera, C. (2015). Música / Cine: variaciones sobre un mismo tema, musicalidad de la imagen o sonata de cuatro movimientos en tono mayor. Luciérnaga, 2 (4), 1-14. Recuperado de: https://revistas.elpoli.edu.co/index. php/luc/article/view/257/224

Bauman, Z. (2002). La cultura como praxis. Barcelona: Paidós.

Bauman, Z. (2007). Vida líquida. Barcelona: Paidós.

Buitrago, F. y Duque, I. (2013). La economía naranja: una oportunidad infinita. Bogotá: Banco Interamericano de Desarrollo.

Capurro, P. (2014). Sin nadie en el medio. El papel de internet como intermediario en las industrias culturales y en la educación. Cuaderno 49 | Centro de Estudios en Diseño y Comunicación (2014), pp 189-207.
Carrasco, C. y Saperas, L. E. (2011). La institucionalización del concepto de industrias culturales en el proceso de debate sobre políticas culturales en la Unesco y el Consejo de Europa (1970- 1982), adComunica. Revista de Estrategias, Tendencias e Innovación en Comunicación, (2)2.

Cassián Yde, N. (2012). De qué está hecha una ciudad creativa. Una propuesta para abordar la cultura, el ocio y la creatividad en la urbe contemporánea. Athenea Digital, 12(1), 169-190.

Castañeda-Rivera, E. y Garduño-Bello, B. (2017). Mapa de las industrias creativas en México. Proyección para CENTRO. Economía Creativa, (7), 118-166.

Castro, S. y Quintero, A. (2018). Oriente sinfónico: procesos de interacción comunicativa en las bandas sinfónicas del Oriente antioqueño. En: Arango, C. Territorio, identidades, comunicación. Rionegro: Fondo Editorial Universidad Católica de Oriente.

Cazau, P. (2006). Introducción a la investigación en ciencias sociales. Buenos Aires: RedPsicología Online.

Chávez Méndez, M. G. (2004). De cuerpo entero... Todo por hablar de música. 
Reflexión técnica y metodológica del grupo de discusión. México: Universidad de Colima.

Cunningham, S. (2011) Las industrias creativas y algunas respuestas a sus críticos. Ekonomiaz (78)3.

Cifuentes, J. C. y Millán, A. M. (2011). Concentración de la producción de las industrias culturales en Cali, Estudios Gerenciales, (27)119.

Ghelfi, D. (2005). El motor de la creatividad en la economía creativa: entrevista a John Howkins. Obtenido de World Intelectual Property Organization: http://www.wipo. int/export/sites/www/ sme/es/documents/pdf/cr_interview_ howkins.pdf.

Martel, F. (2011). Cultura Mainstream. Cómo nacen los fenómenos de masas. Madrid: Taurus.

Manzano, R. (2015). Consumo de jazz contemporáneo en la Habana: el público, el concierto, la experiencia, el gsuto, el conocimiento y la opinión como elementos de mediación y socialización. LUCIERNAGA, 5(9), 92-98. Recuperado de: https://revistas.elpoli.edu.co/index. php/luc/article/view/304/274

Martín Barbero, J. De los medios a las mediaciones: comunicación, cultura y hegemonía. Convenio Andrés Bello, 1998.

Martínez López, J. (2015).Sociedad del entretenimiento (2): Construcción socio - histórica, definición y caracterización de las industrias que pertenecen a este sector. LUCIÉRNAGA, 3(6), 6-16. Recuperado de: https://revistas. elpoli.edu.co/index.php/luc/article/ view/272/238

Montoya, L. E. (2016). Agentes del capital en el discurso de la Planeación en el Altiplano del Oriente Antioqueño. En: Arango, C. Desarrollo y territorio: perspectivas, abordajes, experiencias. Resultados de Investigación. Rionegro: Fondo Editorial Universidad Católica de Oriente, pp. 47-64.
Montoya Restrepo, et al (2016). Estudios culturales y organizacionales: gerencia, cultura y sociedad. Venezuela: Universidad de Carabobo.Disponible en: http://hdl.handle.net/123456789/3737

Navarro Yáñez, C. y Guerrero Panal, G. (2012). Industrias culturales en ciudades españolas. Un primer acercamiento. Revista de Estudios Regionales (94), 71 103.

O'connor, J. (2011). Las industrias creativas y culturales: una historia crítica. Ekonomiaz. Revista vasca de Economía, Gobierno Vasco, (03)78, 24-47.

Prieto Herrero, L. C. (2011). La contribución de la cultura y las artes al desarrollo económico regional. Investigaciones Regionales (19), 177 202.

Jal, R. (2015). Transformando talento en organizaciones sustentables: el desarrollo de emprendimientos de la economía creativa, FACES (21)44, 113123.

Sosnowski, S. (1999). Apuestas culturales al desarrollo integral de América Latina. Trabajo presentado al Foro Desarrollo y Cultura, BID - UNESCO, París, 11 y 12 de Marzo.

Szpilbarg D. y Ezequiel S. (2014). De la industria cultural a las industrias creativas: un análisis de la transformación del término y sus usos contemporáneos. Estudios de Filosofía Práctica e Historia de las Ideas, (16)2.

Valle, M. (2011). Metáfora de la guerra en la construcción sociocultural del clúster textil/confección, diseño y moda de Antioquia. Signo y Pensamiento, 30(58), 280-296. Disponible en : https:// revistas.javeriana.edu.co/index.php/ signoypensamiento/article/view/2499

Wyszomirski, M. (2008). The local creative economy in the United States of America. En: H. Anheier \& Y. Isar (Eds.), The cultural economy: Cultures and globalization. London: Sage.

Zafra, R. (2017). El entusiasmo. Barcelona: Anagrama. 


\section{Notas}

[1] Este artículo es un producto derivado del proyecto de investigación "Oriente imaginado: Exploración del lugar de la comunicación en las representaciones sociales y la identidad. Primera fase", del cual surgen los proyectos "Oriente sonoro: referentes de identidad y territorio en las canciones de las bandas emergentes del Oriente antioqueño", "Oriente visual: referentes de identidad en el videoclip de las bandas emergentes del Oriente antioqueño" y "Mapa de industrias creativas en el Altiplano del Oriente antioqueño", desarrollados por el grupo Communis, financiados por la Dirección de Investigación, Desarrollo e Innovación de la Universidad Católica de Oriente.

\section{Para citar este artículo:}

Arango, C. y Rojas, B. (2019). Comunicación y música. Campos de tensión en la construcción de una industria creativa y cultural en el Oriente Antioqueño- Colombia. Revista Luciérnaga Comunicación. Vol. 11, Núm. 22. Pp. 130 - 145.

Doi: https://doi.org/10.33571/revistaluciernaga.v11 n22a6

OJS. https://revistas.elpoli.edu.co/index.php/luc/issue/archive 